\title{
ON $\pi^{\prime}$-CLOSURE OF $\pi$-HOMOGENEOUS GROUPS
}

\author{
PAMELA FERGUSON
}

\begin{abstract}
Let $\pi$ be a set of odd primes. It is known that $\pi^{\prime}$-closed groups are $\pi$-homogeneous, but that the converse does not hold in general. In this paper we prove that a finite group $G$ which is $D_{\pi}$ and $\pi$-homogeneous is $\pi^{\prime}$-closed.
\end{abstract}

Let $G$ be a finite group, and let $\pi(G)$ denote the set of prime divisors of $|G|$. Let $\pi$ be a subset of $\pi(G)$; then $G$ is $\pi$-homogeneous if $N_{G}(H) / C_{G}(H)$ is a $\pi$-group whenever $H$ is a nontrivial $\pi$-group. Let $\pi^{\prime}=\pi(G)-\pi$; then $G$ is $\pi^{\prime}$-closed if the set of $\pi^{\prime}$-elements forms a subgroup of $G$.

It is known that $\pi^{\prime}$-closed groups are $\pi$-homogeneous. The converse does not hold in general. For example, $A_{5}$ is $5^{\prime}$-homogeneous, but $A_{5}$ is not 5-closed. Thus, some extra conditions are necessary in order that $\pi$ homogeneity implies $\pi^{\prime}$-closure. We shall say that $G$ is a $D_{\pi}$ group if all maximal $\pi$-subgroups of $G$ are conjugate $S_{\pi}$-subgroups. It should be noted that we do not assume all $S_{\pi}$-subgroups of $G$ are solvable. Z. Arad [1] has conjectured that if $\pi$ is a set of primes, then $D_{\pi}$ and $\pi$-homogeneity imply $\pi^{\prime}$-closure. This conjecture is proved in the theorem below.

THEOREM. Let $G$ be a finite group such that $G$ is $D_{\pi}$ and $\pi$-homogeneous, then $G$ is $\pi^{\prime}$-closed.

Throughout this paper we assume $G \in$ Hypothesis A.

Hypothesis A. Let $\pi$ be a set of primes. $G$ is a $D_{\pi} \pi$-homogeneous group of minimal order such that $G$ is not $\pi^{\prime}$-closed.

We will argue by contradiction to show that no such group $G$ ( $G \in$ Hypothesis A) exists.

If $G \in$ Hypothesis A, clearly $\pi(G) \neq \pi$. Let $D$ denote a maximal $\pi$ subgroup of $G$. If $H$ is a subgroup of $G$, and $\tau$ is a set of primes, then let $|H|_{\tau}$ be defined by $|H|=|H|_{\tau} s$ where $(s, \tau)=1$.

The following two results were proved in [2].

Lemma 1 [2, Lemma 2.3]. Subgroups and epimorphic images of $\pi$-homogeneous groups are $\pi$-homogeneous.

LEMMA 2 [2, LEMMA 2.4]. If $K$ is a normal subgroup of the $\pi$-homogeneous group $H$, and if $K$ and $H / K$ are $\pi^{\prime}$-closed, then $H$ is $\pi^{\prime}$-closed.

Lemma 3. Assume $G \in$ Hypothesis A; then $\left|O_{\pi}(G)\right|=1$.

Received by the editors November 16, 1976.

AMS (MOS) subject classifications (1970). Primary 20D20.

O American Mathematical Society 1977 
Proof. We will assume $\left|O_{\pi}(G)\right|>1$ and obtain a contradiction.

Let $\bar{H}$ denote the image of a subset $H \subseteq G$ in $G / O_{\pi}(G)$. Since $G$ is $D_{\pi}$, $O_{\pi}(G) \subseteq D^{g}$ for all $g \in G$. Order arguments imply that the subgroups $\bar{D}^{\overline{8}}$ are $S_{\pi}$ subgroups of $\bar{G}$.

Let $\bar{H}$ be a maximal $\pi$-subgroup of $\bar{G}$. The fundamental homomorphism theorem implies $\left.|H||| G\right|_{\pi}$. Now $G$ a $D_{\pi}$-group implies $H \subseteq D^{g}$ for $g \in G$. Thus $\bar{H} \subseteq \bar{D}^{\bar{g}}$. The maximality of $\bar{H}$ implies $\bar{H}=\bar{D}^{\bar{g}}$. Hence $\bar{G}$ is $D_{\pi}$.

Lemma 1 implies $\bar{G}$ is $\pi$-homogeneous. Now $\left|O_{\pi}(G)\right|>1$ implies $|\bar{G}|<$ $|G|$. Hence $G \in$ Hypothesis A implies $\bar{G}$ is $\pi^{\prime}$-closed. Now $O_{\pi}(G)$ is trivially $\pi^{\prime}$-closed so that Lemma 2 implies $G$ is $\pi^{\prime}$-closed. Thus if $G \in$ Hypothesis A, then $O_{\pi}(G)=1$.

Lemma 4. Assume $G \in$ Hypothesis A and $S$ is a nontrivial p-subgroup of $D$. Then the following conditions hold:

(i) $N_{G}(S)$ is $\pi^{\prime}$-closed;

(ii) $N_{G}(S)=N_{D}(S) O_{\pi^{\prime}}\left(C_{G}(S)\right)$;

(iii) $S \subseteq D^{w}$ implies $D^{w}=D^{y}$ where $y \in O_{\pi^{\prime}}\left(C_{G}(S)\right)$.

Proof. Lemma 1 implies $N_{G}(S)$ is $\pi$-homogeneous and Lemma 3 implies $\left|N_{G}(S)\right|<|G|$. Hence, in order to show that $N_{G}(S)$ is $\pi^{\prime}$-closed it is sufficient to show that $N_{G}(S)$ is $D_{\pi}$.

Suppose $K$ is a maximal $\pi$ subgroup of $N_{G}(S)$. Clearly $K \supseteq S$. Since $G$ is $D_{\pi}, K \subseteq D^{g}$ for some $g \in G$. Hence $K=N_{D^{8}}(S)$ where $S \subseteq D^{g}$.

Let $p^{n}=|D|_{p}$. We will proceed by induction on $p^{n} /|S|$. If $p^{n} /|S|=1$, then $S$ is a Sylow $p$-subgroup of $D$. Suppose that $S \subseteq D^{w} \cap D$. Sylow's theorems imply $S=S^{d w}$ where $d \in D$. Hence

$$
N_{D^{w}}(S)=N_{D^{w}}\left(S^{d w}\right)=N_{D^{d w}}\left(S^{d w}\right)=\left(N_{D}(S)\right)^{d w} .
$$

Now $d w \in N_{G}(S)$ implies

$$
N_{D^{w}}(S)=\left(N_{D}(S)\right)^{r} \quad \text { where } r \in N_{G}(S) .
$$

Now let $K$ be a maximal $\pi$-subgroup of $N_{G}(S)$; then $K=N_{D^{*}}(S)$ where $S \subseteq D^{w}$. Thus (*) implies $K$ is conjugate in $N_{G}(S)$ to $N_{D}(S)$. In particular, $N_{D}(S)$ is a maximal $\pi$-subgroup of $N_{G}(S)$. Moreover, all maximal $\pi$ subgroups are conjugate in $N_{G}(S)$ to $N_{D}(S)$. It follows easily that $N_{G}(S)$ is $D_{\pi}$. Hence, $N_{G}(S)$ is $\pi^{\prime}$-closed. Since $N_{G}(S)$ is $\pi$-homogeneous, we see that $O_{\pi^{\prime}}\left(N_{G}(S)\right)=O_{\pi^{\prime}}\left(C_{G}(S)\right)$. Order arguments now imply $N_{G}(S)=$ $N_{D}(S) O_{\pi^{\prime}}\left(C_{G}(S)\right)$.

If $S \subseteq D^{w}$, then $S=S^{d w}$ for $d \in D$ implies $d w \in N_{G}(S)$. Thus, $d w=d_{2} y$ where $d_{2} \in N_{D}(S)$ and $y \in O_{\pi^{\prime}}\left(C_{G}(S)\right)$. Hence $D^{w}=D^{y}$.

Now suppose $p^{n}>p^{n} /|S|>1$ and the lemma is true for all $T$ such that $p^{n} /|T|<p^{n} /|S|$ (i.e., $\left.|T|>|S|\right)$.

Let $S_{1}$ be a Sylow $p$-subgroup of $N_{G}(S)$, and let $K$ be a maximal $\pi$ subgroup of $N_{G}(S)$ such that $S_{1} \subseteq K$. Then $K=N_{D^{\prime}}(S)$ where $S \subseteq D^{f}$. Now $S$ is not a Sylow $p$-subgroup of $D$. Hence Sylow's theorems imply if $T$ is a Sylow $p$-subgroup of $N_{D}(S)$, then $|T|>|S|$. Moreover, $T \subseteq S_{1}^{r}$ where $r \in$ 
$N_{G}(S)$. Thus $T \subseteq K^{r} \cap D \subseteq D^{f r} \cap D$. Since $|T|>|S|, D^{f r}=D^{y}$ where $y \in O_{\pi^{\prime}}\left(C_{G}(T)\right)$. Hence, $D^{f}=D^{y r^{-1}}$, which implies

$$
N_{D^{f}}(S)=N_{D^{y-1}}(S)=\left(N_{D}\left(S^{n^{-1}}\right)\right)^{y r^{-1}} .
$$

However, $S \subseteq T$ implies $y \in C_{G}(S)$. Hence, $N_{D^{\prime}}(S)=\left(N_{D}(S)\right)^{y r^{-1}}$ where $y r^{-1} \in N_{G}(S)$. Thus $N_{D^{\prime}}(S)$ is conjugate to $N_{D}(S)$ in $N_{G}(S)$. In particular, $N_{D}(S)$ is a maximal $\pi$-subgroup of $N_{G}(S)$ and $T$ is a Sylow $p$-subgroup of $N_{G}(S)$.

Suppose $S \subseteq D^{w}$; then $S$ is not a Sylow $p$-subgroup of $D^{w}$. Hence if $V$ is a Sylow $p$-subgroup of $N_{D^{w}}(S)$, then $|V / S| \geqslant p$. Sylow's theorems imply $V \subseteq T^{r}$ where $r \in N_{G}(S)$. Thus $V=T_{1}^{r}$ where $T_{1} \subseteq T$ and $T_{1} \supset S$. Hence $T_{1}=V^{r^{-1}} \subseteq D^{w r^{-1}} \cap D$. Now $\left|T_{1}\right|>|S|$ implies $D^{w r^{-1}}=D^{x}$ where $x \in$ $O_{\pi^{\prime}}\left(C_{G}\left(T_{1}\right)\right)$. Hence $x \in C_{G}(S)$, so that $D^{w}=D^{x r}$, where $x r \in N_{G}(S)$. Thus

$$
N_{D^{w}}(S)=N_{D^{x r}}(S)=\left(N_{D}\left(S^{r^{-1} x^{-1}}\right)\right)^{x r}=\left(N_{D}(S)\right)^{x r} .
$$

Thus $N_{D^{w}}(S)$ is conjugate in $N_{G}(S)$ to $N_{D}(S)$. In particular, $N_{D^{w}}(S)$ is a maximal $\pi$-subgroup of $N_{G}(S)$. If $K$ is any maximal $\pi$-subgroup of $N_{G}(S)$, then $K=N_{D^{w}}(S)$ for some $D^{w} \supseteq S$. Hence $K=\left(N_{D}(S)\right)^{z}$, where $z \in$ $N_{G}(S)$. It follows that $N_{G}(S)$ is $D_{\pi}$. Lemmas 1 and 3 imply $N_{G}(S)$ is $\pi^{\prime}$-closed. Now $O_{\pi^{\prime}}\left(N_{G}(S)\right)=O_{\pi^{\prime}}\left(C_{G}(S)\right)$, and order arguments imply $N_{G}(S)$ $=N_{D}(S) O_{\pi^{\prime}}\left(C_{G}(S)\right)$.

If $S \subseteq D^{w}$, then the previous paragraph implies $N_{D^{w}}(S)$ is a maximal $\pi$-subgroup of $N_{G}(S)$. Since $N_{G}(S)=N_{D}(S) O_{\pi^{\prime}}\left(C_{G}(S)\right), \quad N_{D^{w}}(S)=$ $\left(N_{D}(S)\right)^{x}$ for $x \in O_{\pi^{\prime}}\left(C_{G}(S)\right)$. Thus $D^{w x^{-1}} \cap D \supseteq T$. Now $|T|>|S|$ implies $D^{w x^{-1}}=D^{u}$ where $u \in O_{\pi^{\prime}}\left(C_{G}(T)\right)$. Thus $D^{w}=D^{y}$ where $y=u x$ clearly is contained in $O_{\pi^{\prime}}\left(C_{G}(S)\right)$. The lemma follows by induction.

Lemma 5. Assume $G \in$ Hypothesis A. Let $z \in D^{\#}$; then $C_{G}(z)$ is $\pi^{\prime}$-closed. Moreover, $z \in D^{w}$ implies $D^{w}=D^{y}$ where $y \in O_{\pi^{\prime}}\left(C_{G}(z)\right)$.

Proof. Let $z$ be an element of minimal order such that the lemma fails. Suppose $|\langle z\rangle|=p^{k}$ where $p$ is a prime. Lemma 4 implies a contradiction. Thus we may assume $z=z_{1} z_{2}$ where $\left.\left|\left\langle z_{1}\right\rangle\right|=p^{n}\right\rangle 1,\left(\left|\left\langle z_{2}\right\rangle\right|, p\right)=1$, and $z_{2} \neq 1$. Clearly $C_{\tilde{s}}(z)=C_{G}\left(z_{1}\right) \cap C_{G}\left(z_{2}\right)$. Since $1<\left|\left\langle z_{i}\right\rangle\right|\langle|\langle z\rangle|$ for $i=1$, $2, C_{G}\left(z_{i}\right)$ is $\pi^{\prime}$-closed for $i=1,2$. Hence $C_{G}(z)$ is $\pi^{\prime}$-closed.

Now $z \in D \cap D^{w}$ implies $z_{i} \in D \cap D^{w}$ for $i=1$, 2. Lemma 4 implies $D^{w}=D^{y}$ where $y \in O_{\pi^{\prime}}\left(C_{G}\left(z_{1}\right)\right)$. Hence, $z_{2} \in D^{y}=D^{w}$ which implies $z_{2}^{y^{-1}}$ $\in D$. Thus

$$
\left[z_{2}, y^{-1}\right]=z_{2}^{-1} z_{2}^{y^{-1}} \in D \cap O_{\pi^{\prime}}\left(C_{G}\left(z_{1}\right)\right)=1
$$

Therefore,

$$
y \in O_{\pi^{\prime}}\left(C_{G}\left(z_{1}\right)\right) \cap O_{\pi^{\prime}}\left(C_{G}\left(z_{2}\right)\right)=O_{\pi^{\prime}}\left(C_{G}(z)\right) .
$$

Lemma 6. Assume $G \in$ Hypothesis A. Any two elements of $D^{\#}$ which are conjugate in $G$ are also conjugate in $D$. 
Proof. Suppose $z_{1}=z_{2}^{w}$ where $z_{1}, z_{2} \in D^{\#}$. Then $z_{1} \in D \cap D^{w}$. Lemma 5 implies $D^{w}=D^{y}$ where $y \in O_{\pi^{\prime}}\left(C_{G}\left(z_{1}\right)\right)$. Hence $w y^{-1} \in N_{G}(D)$. Since $D$ is a Hall $\pi$-subgroup of $G,\left.\left|N_{G}(D) / D\right||| G\right|_{\pi^{\prime}}$. Theorem 6.2.1 [5] implies $N_{G}(D)$ $=M D$ where $M$ is a $\pi^{\prime}$-subgroup of $N_{G}(D)$. Since $N_{G}(D)$ is $\pi$-homogeneous we see that $M \subseteq C_{G}(D)$. Hence, $N_{G}(D)=M \times D$. Now $w y^{-1} \in N_{G}(D)$ implies $w=d u y^{+1}$ where $u \in M$. Thus $z_{1}=z_{2}^{w}$ implies $z_{2}=z_{1}^{y^{-1} u^{-1} d^{-1}}=$ $z_{1}^{d^{-1}}$. Thus $z_{1}$ is conjugate to $z_{2}$ in $D$.

Proof of Theorem. In order to prove the Theorem, we will show that $G$ satisfies the hypothesis of Theorem 8.22 of [6] with $H=D$. Let $E$ be an elementary subgroup of $G$ such that $|E||| D \mid$. Since $G$ is $D_{\pi}, E \subseteq D^{g}$ for some $g \in G$. Hence $E$ is conjugate to a subgroup of $D$. Lemma 6 implies whenever two elements of $D$ are conjugate in $G$, then they are conjugate in $D$. Theorem 8.22 [6] now implies $G$ is $\pi^{\prime}$-closed.

\section{REFERENCES}

1. Z. Arad, $\pi$-homogeneity and $\pi^{\prime}$-closure of finite groups, Pacific J. Math. 51 (1974), 1-9.

2. R. Baer, Closure and dispersion of finite groups, Illinois J. Math. 2 (1958), 619-640.

3. W. Feit, On a conjecture of Frobenius, Proc. Amer. Math. Soc. 7 (1956), 177-187.

4. __ Characters of finite groups, Benjamin, New York, 1967.

5. D. Gorenstein, Finite groups, Harper and Row, New York, 1968.

6. M. Isaacs, Character theory of finite groups, Academic Press, New York, 1976.

Department of Mathematics, University of Miami, Coral Gables, Florida 33124 